\title{
An extremely wide and very low-mass pair with common proper motion $\star$
}

\section{Is it representative of a nearby halo stream?}

\author{
R.-D. Scholz ${ }^{1}$, N. V. Kharchenko ${ }^{1,2}$, N. Lodieu ${ }^{3}$, and M. J. McCaughrean ${ }^{4}$ \\ 1 Astrophysikalisches Institut Potsdam, An der Sternwarte 16, 14482 Potsdam, Germany \\ e-mail: [rdscholz;nkharchenko] @aip.de \\ 2 Main Astronomical Observatory, 27 Akademika Zabolotnogo St., 03680 Kiev, Ukraine \\ e-mail: nkhar@mao.kiev.ua \\ 3 Instituto de Astrofisica de Canarias, C/ Via Lactea s/n, 38205 La Laguna, Tenerife, Spain \\ e-mail: nlodieu@iac.es \\ 4 Astrophysics Group, School of Physics, University of Exeter, Stocker Road, Exeter EX4 4QL, UK \\ e-mail: mjm@astro.ex.ac.uk
}

Received 18 March 2008 / Accepted 18 June 2008

ABSTRACT

\begin{abstract}
Aims. We describe the discovery of an extremely wide pair of low-mass stars with a common large proper motion and discuss their possible membership in a Galactic halo stream crossing the Solar neighbourhood.

Methods. In a high proper motion survey of the southern sky we used multi-epoch positions and photometry from the SuperCOSMOS Sky Surveys. New nearby ultracool dwarf and subdwarf candidates were selected among the faint and red high proper motion objects, and subsequently confirmed by low-resolution classification spectroscopy. The resulting spectroscopic distance estimates, approximate radial velocity measurements and improved proper motions involving additional epochs from the Two Micron All Sky Survey and from the DEep Near-Infrared Survey were used to compute Galactic space velocities.

Results. The late-type (M7) dwarf SSSPM J2003-4433 and the ultracool subdwarf SSSPM J1930-4311 (sdM7) sharing the same very large proper motion of about $860 \mathrm{mas} / \mathrm{yr}$ were found in the same sky region with an angular separation of about $6^{\circ}$. From the comparison with other high proper motion catalogues we have estimated the probability of a chance alignment of the two new large proper motions to be less than $0.3 \%$. From the individually estimated spectroscopic distances of about $38_{-7}^{+10} \mathrm{pc}$ and $72_{-16}^{+21} \mathrm{pc}$, respectively for the M7 dwarf and the sdM 7 subdwarf, and in view of the accurate agreement in their large proper motions we assume a common distance of about $50 \mathrm{pc}$ and a projected physical separation of about $5 \mathrm{pc}$. The mean heliocentric space velocity of the pair $(U, V, W)=(-232,-170,+74) \mathrm{km} \mathrm{s}^{-1}$, based on the correctness of the preliminary radial velocity measurement for only one of the components and on the assumption of a common distance and velocity vector, is typical of the Galactic halo population.

Conclusions. The large separation and the different metallicities of dwarfs and subdwarfs make a common formation scenario as a wide binary (later disrupted) improbable, although there remains some uncertainty in the spectroscopic classification scheme of ultracool dwarfs/subdwarfs so that a dissolved binary origin cannot be fully ruled out yet. It seems more likely that this wide pair is part of an old halo stream. Higher-resolution spectroscopic observations are needed to measure accurate radial velocities of both components. Further, we suggest to check the M7 dwarf for an unresolved binary status, which would explain its shorter spectroscopic distance estimate, and to place both objects on a trigonometric parallax program.
\end{abstract}

Key words. stars: kinematics - stars: low-mass, brown dwarfs - Galaxy: formation - Galaxy: halo - solar neighbourhood

\section{Introduction}

Common proper motion (CPM) stars are typically wide binary stars for which the measuring errors of the proper motion are much larger than the effect of orbital motion. Wide binaries of different masses are interesting for stellar evolution studies since their components, believed to have the same age and metallicity, have evolved without interaction and can be studied independently and compared to each other (e.g. Seifahrt et al. 2005; Catalán et al. 2008). Halo wide binaries provide important traces of the formation of the Galaxy (Allen et al. 2007) and can be used to constrain Galactic halo dark matter (Chanamé \& Gould 2004).

* Based on observations with the ESO $3.6 \mathrm{~m} / \mathrm{EFOSC} 2$ at the European Southern Observatory, La Silla (ESO program 70.C-0568).
CPM stars are usually found among stars with large proper motions where the compatibility of the proper motions is easier to detect. The ratio between the angular separation and the proper motion can be used as a criterion to discriminate between physical and optical pairs (Halbwachs 1986). But even for very large separations, a physical connection in terms of membership in a cluster or stellar stream (or a dissolved binary status) is obvious if the individual proper motions are much larger than their measuring errors and agree within these errors.

The LDS (Luyten Double Stars) catalogue of CPM stars (Luyten 1940-1987) lists 20 pairs with angular separations larger than 600 arcsec (maximum 9000 arcsec). Only four of these pairs have a proper motion above the lower limit of the Luyten Half Second (LHS; Luyten 1979a) catalogue of $500 \mathrm{mas} / \mathrm{yr}$. For LDS entries with smaller proper motions and 
relatively large separations there is a higher probability of random pairings (see e.g. LDS 4041 and LDS 4999 in Scholz et al. 2005). The catalogue of wide binaries by Allen et al. (2000) includes a few very wide CPM pairs with angular separations up to about 800 arcsec and with a maximum expected semi-major axis of about $125000 \mathrm{AU}$. At least the primary components are typically bright Hipparcos stars so that the projected physical separations given in that catalogue rely on trigonometric parallax measurements.

Extremely wide (angular separation $>1^{\circ}$ ) binaries and multiple systems with pre-Hipparcos trigonometric parallaxes were presented in Gliese \& Jahreiß (1988). In their list of 32 systems with projected tangential separations exceeding $5000 \mathrm{AU}$, there are 9 systems with angular separations between $1^{\circ}$ and $16^{\circ}$, corresponding to minimum physical separations of $10000 \mathrm{AU}$ up to more than a million AU (their proper motions range between 0.2 and 3.8 arcsec/yr). Four of the latter were discussed by the authors as gravitationally unbound due to their large separations and differences in their parallaxes or radial velocities. The Hipparcos parallaxes (ESA 1997) have shown that only 3 out of the above mentioned 9 systems, Gl 881+879, Gl 803+799AB, and $\mathrm{Gl} 559 \mathrm{AB}+551$, can be considered as physical since their parallaxes agree to within 5\% and their projected linear separations are 54250, 47180 , and $10380 \mathrm{AU}$, respectively ${ }^{1}$. It is remarkable that for the first two of these systems, which are located at distances of 8 and $10 \mathrm{pc}$, respectively, all proper motion components do also agree to within about $10 \mathrm{mas} / \mathrm{yr}$. This is not the case for $\mathrm{Gl} 559 \mathrm{AB}+551(=\alpha \mathrm{CenAB}+$ Proxima $)$, where the distance of only $1.3 \mathrm{pc}$ and the orbital motions result in differences up to a few $100 \mathrm{mas} / \mathrm{yr}$ between the three components of this bound system (Wertheimer \& Laughlin 2006).

All previously known extremely wide systems include at least one star with $>0.5 M_{\odot}$. In this paper, we present the first extremely wide CPM pair consisting of two very low-mass $\left(\approx 0.1 M_{\odot}\right)$ stars. In Sect. 2 we describe the astrometric measurements and show how accurately the large proper motions of the two faint red stars separated by about $6^{\circ}$ agree with each other. In Sect. 4 we present a rather surprisingly different spectroscopic classification of the components of this extremely wide CPM pair, and in Sect. 5 we discuss their possible membership in a Galactic halo stream.

\section{Common proper motion}

The two high proper motion (HPM) objects SSSPM J1930-4311 (hereafter SSSPM 1930) and SSSPM J2003-4433 (hereafter SSSPM 2003) were discovered in the course of a HPM survey (described in Lodieu et al. 2005) using multi-epoch SuperCOSMOS Sky Surveys (SSS) data (Hambly et al. 2001). The 3rd search strategy outlined in Lodieu et al. (2005; Sect. 2) was applied, i.e. only $R$ and $I$ band data were used for the HPM detection. The proper motion solution for both objects was later improved by including all available SSS data as well as the positions measured in the Two-Micron All Sky Survey (2MASS; Cutri et al. 2003), in the DEep Near-Infrared Survey (DENIS; Epchtein et al. 1997), using the third DENIS data release from 2005, and the positions of our targets on the acquisition images taken with the ESO $3.6 \mathrm{~m}$ telescope. The latter were measured

1 Three other systems (Gl 331ABC+332AB, Gl 48+22AB, Gl $1255 \mathrm{ABC}+\mathrm{HD}$ 199476) are questionable physical systems with projected linear separations between 100000 and $350000 \mathrm{AU}$ and parallax differences of $10-15 \%$.
Table 1. Astrometry and photometry of SSSPM 1930.

\begin{tabular}{|c|c|c|c|}
\hline$\alpha, \delta(\mathrm{J} 2000.0)\left[\mathrm{h} \mathrm{m} \mathrm{s}^{\circ}{ }^{\prime}{ }^{\prime \prime}\right]$ & Epoch & Magnitude & Data \\
\hline $192941.019-43 \quad 1017.73$ & 1978.675 & $B_{J}=21.077$ & SSS-UK \\
\hline $192941.036-431017.96$ & & & corr. \\
\hline $192941.033-431025.42$ & 1987.657 & $R=18.315$ & SSS-ESO \\
\hline $192941.014-431025.63$ & & & corr. \\
\hline $192940.986-431028.64$ & 1991.543 & $I=16.311$ & SSS-UK \\
\hline $192941.009-431028.89$ & & & corr. \\
\hline $192940.987-431032.24$ & 1995.630 & $R=18.531$ & SSS-UK \\
\hline $192941.005-431032.56$ & & & corr \\
\hline $192941.01 \quad-43 \quad 1033.0$ & 1996.416 & $I=16.261$ & DENIS \\
\hline $192940.999-431033.24$ & & & corr \\
\hline \multirow[t]{3}{*}{$192940.99-43$} & 2000.633 & $J=14.794$ & 2MASS \\
\hline & & 4.230 & 2MASS \\
\hline & & 4.091 & 2MASS \\
\hline $192941.01-431037.6$ & 2000.503 & $I=16.267$ & DENIS \\
\hline $192941.003-431037.61$ & 2001.499 & & corr. \\
\hline $192941.002-431038.83$ & 2002.934 & & $3.6 \mathrm{~m}$ \\
\hline
\end{tabular}

Table 2. Astrometry and photometry of SSSPM 2003.

\begin{tabular}{|c|c|c|c|}
\hline$\alpha, \delta(\mathrm{J} 2000.0)\left[\mathrm{h} \mathrm{m} \mathrm{s}^{\circ}{ }^{\prime}{ }^{\prime \prime}\right]$ & Epoch & Magnitude & Data \\
\hline $200252.096-443247.01$ & 1980.392 & $B_{J} \approx 22$ & SSS-UK \\
\hline $200252.116-443247.21$ & & & corr. \\
\hline $200252.097-443253.57$ & 1988.448 & $R=19.094$ & SSS-ESO \\
\hline $2.089-443253.93$ & & & corr \\
\hline $200252.074-443258.90$ & 1994.364 & $I=16.075$ & SSS-UK \\
\hline $200252.094-443259.09$ & & & corr. \\
\hline $200252.079-443259.65$ & 1995.409 & $R=19.564$ & SSS-UK \\
\hline $200252.097-443259.88$ & & & corr \\
\hline \multirow[t]{3}{*}{$200252.08-443303.6$} & 1999.627 & $J=13.528$ & 2MASS \\
\hline & & 2.987 & 2MASS \\
\hline & & 86 & 2MASS \\
\hline $200252.06-$ & 1998.701 & $I=15.957$ & DENIS \\
\hline $200252.099-443304.34$ & 2000.458 & & cor \\
\hline $200252.054-443306.42$ & 2002.932 & & $3.6 \mathrm{~m}$ \\
\hline
\end{tabular}

by us using the $2 \mathrm{MASS}$ positions of 15 reference stars with no significant proper motion in the SSS database.

The multi-epoch positions used for the proper motion determinations as well as the photometry are listed in Tables 1 and 2. Note that for SSSPM 2003 there was no original SSS measurement on the $B_{J}$ plate since the object was merged with a background star which appeared isolated and without a significant proper motion on all other SSS plates. We measured the position of SSSPM 2003, overlapping with the background star in east-west direction on the $B_{J}$ plate, by eye.

The proper motion of the two HPM objects as determined from the original SSS data alone (run \#1 in Table 3) was already in relatively good agreement so that we started to think about a possible connection between them. After including all the additional epochs (run \#2) we got an even better agreement, but the errors remained very large. The main reason was that for each object one DENIS position was off by about an arcsecond. We checked the data taken from the VizieR Catalogue Service (http://vizier.u-strasbg.fr/), downloaded the corresponding DENIS images, and found a discrepancy between the epochs given in the catalogue and in the FITS headers of the images. We preferred the information from the FITS header which was also consistent with the DENIS strip number given in the catalogue. After correcting two DENIS epochs the proper motion errors in run \#3 decreased significantly.

We tried to further improve the proper motion determination by correcting all the different epoch astrometry relative to the 
Table 3. Proper motion $\left(\mu_{\alpha} \cos \delta, \mu_{\delta}\right)$ solutions [mas/yr].

\begin{tabular}{ccc}
\hline \hline$\#$ & SSSPM 1930 & \multicolumn{2}{c}{ SSSPM 2003 } \\
\hline 1 & $-24.1 \pm 18.4,-853.7 \pm 4.8$ & $-32.3 \pm 11.7,-882.8 \pm 23.2$ \\
2 & $-11.0 \pm 8.1,-883.5 \pm 17.9$ & $-19.4 \pm 5.4,-879.7 \pm 25.8$ \\
3 & $-10.5 \pm 8.0,-871.8 \pm 5.8$ & $-19.3 \pm 5.0,-859.2 \pm 11.7$ \\
4 & $-16.4 \pm 3.1,-861.6 \pm 2.7$ & $-18.0 \pm 7.4,-855.2 \pm 3.8$ \\
\hline
\end{tabular}

Notes. \#1 Based on the original 4 SSS epochs for SSSPM 1930 and 3 SSS epochs (without $B_{J}$ ) for SSSPM 2003; \#2 with all the uncorrected positions from Table 1 ( 8 epochs) and Table 2 (7 epochs), respectively; \#3 after correcting two DENIS epochs; \#4 finally adopted run: with corrected DENIS epochs and after transforming all SSS and DENIS positions to the 2MASS system.

2MASS system. The same 15 reference stars, the 2MASS positions of which were already used for calibrating the astrometry on the acquisition images, served for a new measurement of our target positions on the SSS images and for correcting the DENIS catalogue positions (all lines with corrected data in Tables 1 and 2). The resulting proper motion solutions (run \#4 in Table 3) are again much more accurate. We adopt this run as our final solution. It is remarkable that the final results for SSSPM 1930 and SSSPM 2003 are in excellent agreement within the errors.

\section{Probability of a chance alignment of the CPM pair}

Chance alignments of CPM stars have been recently investigated by Lépine \& Bongiorno (2007) in a search for new distant companions to nearby stars. They looked for faint wide CPM companions to Hipparcos (ESA 1997) stars in the new Lépine-Shara Proper Motion-North catalogue (LSPM-North; Lépine \& Shara 2005). Studying the proper motion distribution in the LSPM-North and simulating chance alignments between the two catalogues, they found that real CPM doubles satisfy the condition $\Delta \theta \Delta \mu<(\mu / 0.15)^{3.8}$, where $\Delta \theta$ is the angular separation (in arcsec), $\Delta \mu$ is the magnitude of the difference between the proper motion vectors (in $\operatorname{arcsec} / y r$ ), and $\mu$ is the mean total proper motion of the pair (in $\operatorname{arcsec} / \mathrm{yr}$ ). The range of separations they used in their search $(\Delta \theta<1500$ arcsec $)$ is much smaller than in our case $(\Delta \theta \approx 21600 \operatorname{arcsec})$. However, the large mean proper motion $(\mu=0.86 \mathrm{arcsec} / \mathrm{yr})$ and the very small proper motion difference $(\Delta \mu<0.015 \mathrm{arcsec} / \mathrm{yr})$ of SSSPM 1930 and SSSPM 2003 compensate for the wide separation so that the above condition is fulfilled.

In order to compute the probability of a chance alignment of our CPM pair we have compared its proper motions and distribution on the sky with known HPM stars from the New Luyten Two Tenths catalogue (NLTT; Luyten 1979b). Figure 1 shows the central part of the NLTT proper motion diagram. There are only 46 NLTT stars falling between an upper limit of $0.865 \mathrm{arcsec} / \mathrm{yr}$ and a lower limit of $0.850 \mathrm{arcsecs} / \mathrm{yr}$ in the total proper motion (i.e. in the region between the two large circles). The proper motion limits were selected taking into account the proper motion errors of the two components of our pair. These are conservative limits, since their total proper motions agree in fact within $0.007 \mathrm{arcsec} / \mathrm{yr}$. Their position angles agree within about 0.1 so that there is only one line seen in Fig. 1 for the position angles of the two proper motions.

Figure 2 shows the distribution of all NLTT stars with $\mu>$ $0.18 \mathrm{arcsec} / \mathrm{yr}$ on the sky, with the 46 stars selected from the previous proper motion diagram marked again by small filled circles. Whereas the incompleteness of the NLTT in the Galactic plane and with $\delta<-30^{\circ}$ is clearly seen, the selected stars with

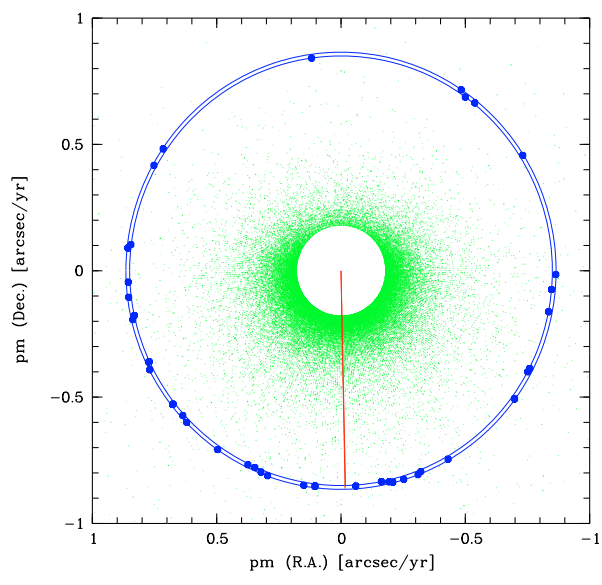

Fig. 1. Distribution of NLTT proper motions. Dots show the NLTT stars with $\mu>0.18$ arcsec/yr and proper motion components of less than \pm 1 arcsec/yr. The two large circles show proper motion limits of 0.850 and 0.865 arcsec/yr. Small filled circles mark the NLTT stars falling between these proper motion limits. The straight line shows the position angles of the proper motions of SSSPM 1930/SSSPM 2003.

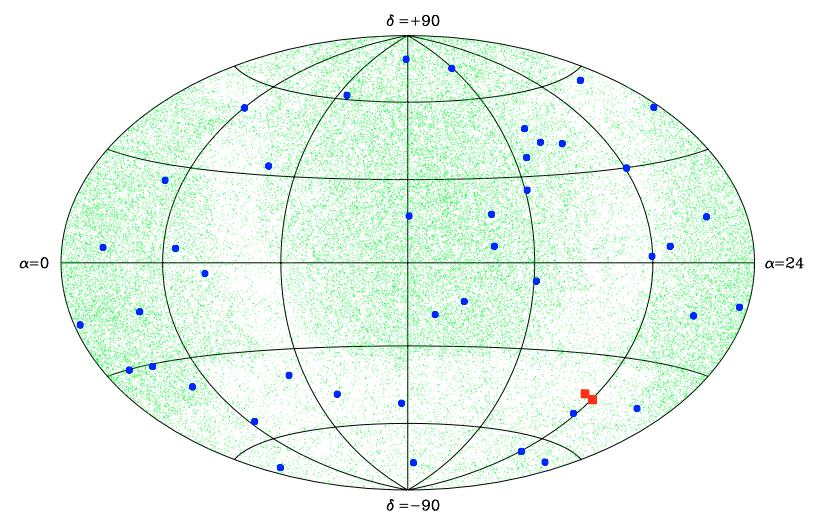

Fig. 2. Distribution of all NLTT stars with $\mu>0.18 \operatorname{arcsec} / \mathrm{yr}$ on the sky. Small filled circles mark the NLTT stars with proper motions between 0.850 and 0.865 arcsec/yr. The two filled squares mark the positions of SSSPM 1930 and SSSPM 2003.

relatively large proper motions seem to be randomly distributed. With the given density of 46 stars over the 41253 square degrees of the full sky we compute the probability that two of these stars are separated by only $6^{\circ}$ as $46 /(41253 / 36 \pi)=0.126$, which does not yet take into account the position angles of the proper motions. The probability that two of the 46 stars located in the $360^{\circ}$ ring in Fig. 1 have a very small $\left(0^{\circ} .1\right)$ difference in the proper motion orientation is only $46 /(360 / 0.1)=0.0128$, which does however not take into account the asymmetrical distribution of the proper motions in declination. Using only 37 stars with the corresponding negative proper motion components (half ring of $180^{\circ}$ ), we derive a somewhat higher probability of $37 /(180 / 0.1)=0.0206$. The final probability of a chance alignment in both proper motions and position angles can then be derived as $0.126 \times 0.0206=0.0026$, which is small enough to rule out a random pairing of SSSPM 1930 and SSSPM 2003.

\section{Spectroscopic distance estimates}

Spectroscopic follow-up observations of both components of the extremely wide CPM pair were carried out with the ESO $3.6 \mathrm{~m}$ telescope at La Silla between December 5th and 8th, 2002 using the EFOSC2 instrument. Based on their red colour, both 


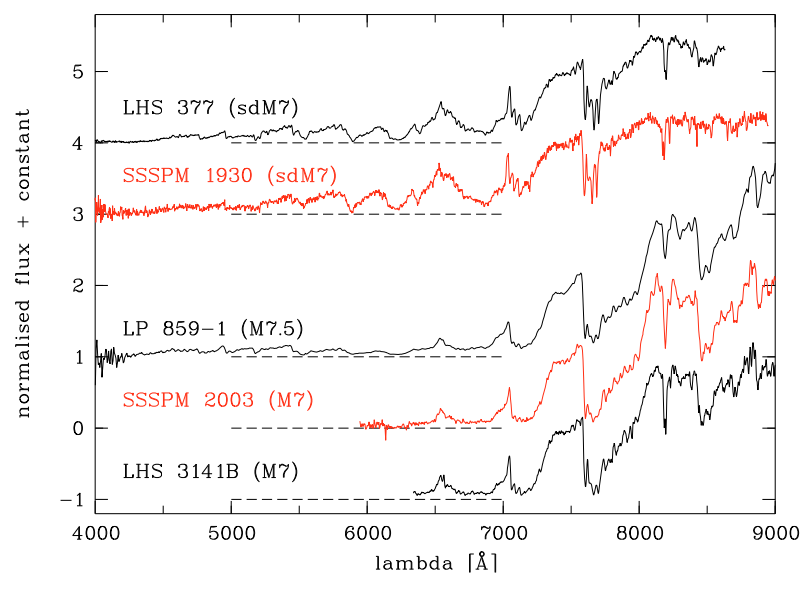

Fig. 3. Low-resolution classification spectra of SSSPM 1930, SSSPM 2003 and some comparison objects. The ESO NTT/EMMI spectrum of SSSPM 1930 is from Scholz et al. (2004), the Keck/LRIS spectrum of LHS 377 was taken from Neill Reid's Web page (http://dept.physics. upenn. edu/ inr/ultracool.html). The ESO $3.6 \mathrm{~m} / \mathrm{EFOSC} 2$ spectrum of SSSPM 2003 is shown together with ESO VLT/FORS1 spectra of two comparison objects from Lodieu et al. (2005): LP 859-1 and LHS 3141B. All spectra are flux-calibrated and normalised at $7500 \AA$.

HPM objects were initially treated by us as independent candidates for previously unknown ultracool (M- and L-type) stars and brown dwarfs in the immediate Solar neighbourhood. The observations and data reductions have already been described in Lodieu et al. (2005). For details of the applied spectroscopic classification scheme we refer the reader to that paper.

As already mentioned by Lodieu et al. (2005), the observing conditions during that particular run were not ideal. Therefore, only a rather noisy spectrum was obtained for SSSPM 1930. Despite the low signal-to-noise ratio of that spectrum (not shown here) we were able to establish a late subdwarf status with a preliminary spectral type of sdM5.5. A much higher-quality spectrum of SSSPM 1930 was observed by Scholz et al. (2004) with the ESO NTT equipped with the EMMI instrument (see Fig. 3). It is shown together with the spectrum of LHS 377, the latesttype (sdM 7) object found by Gizis (1997) according to his classification scheme for late-K and $M$ subdwarfs. Based on the spectral indices used in this scheme, Scholz et al. (2004) found also an sdM 7 spectral type for SSSPM 1930.

The second component of the pair, SSSPM 2003, turned out to be a normal late-type $\mathrm{M}$ dwarf according to our ESO 3.6 m/EFOSC2 spectrum (Fig. 3). However, the spectral indices measured for SSSPM 2003 lead to rather different spectral types of M 6.1 (from TiO5 = 0.195 and $\mathrm{VOa}=2.129$ ) and M 7.5 (from PC3 = 1.725; indices as used by Lodieu et al. 2005). About one third of the M 6-M 8 dwarfs in Lodieu et al. (2005) do also show systematically later spectral types if defined by the PC3 index (see their Fig. 8). The two comparison objects LHS 3141B (M7) and LP 859-1 (M 7.5), also shown in Fig. 3, did not have these classification problems in Lodieu et al. (2005). The latter was also found to be an M7.5 dwarf by Gizis et al. (2000) and Reid et al. (2002). We adopt a spectral type of M 7 for SSSPM 2003 with the relatively large uncertainty of 1.0 subtypes, taking into account the different typing by spectral indices as well as the visual comparison with template spectra.

With the above classification, and using the absolute $J$ magnitudes of M 6-M 8 dwarfs from Scholz, Meusinger \& Jahreiß (2005), we estimate the distance of SSSPM 2003 as $38_{-7}^{+10}$ pc.
On the other hand, we use the 2MASS $J H K_{\mathrm{S}}$ magnitudes of LHS 377 and its trigonometric parallax listed in Gizis (1997) for deriving a distance of $72_{-16}^{+21}$ pc for SSSPM 1930, conservatively assuming an uncertainty of 0.5 in the absolute magnitudes of these poorly investigated ultracool (sdM7) subdwarfs. If we further assume our CPM objects, the large proper motions of which agree to within $1 \%$, to be at approximately the same distance from the Sun, we may expect a common distance of roughly $50_{-15}^{+25} \mathrm{pc}$. In the following we discuss this common distance assumption with respect to an expected common space velocity.

\section{Discussion - halo stream and metallicity}

The assumed distance interval leads to projected physical separations between 3.5 and 8 pc so that we can rule out a wide binary. Therefore, we investigate the kinematic connection between the two objects as possible members in a stellar stream.

The proper motion error of less than 10 mas/yr corresponds with the assumed distance of $50 \mathrm{pc}$ to an error in the tangential velocity of less than $2 \mathrm{~km} \mathrm{~s}^{-1}$. However, the large uncertainty in the distance to our pair translates with a proper motion of $860 \mathrm{mas} / \mathrm{yr}$ to a wide tangential velocity interval of $204_{-61}^{+102} \mathrm{~km} \mathrm{~s}^{-1}$. A radial velocity $(R V)$ measurement is available for only one of the components, SSSPM 1930, with $-262 \pm$ $25 \mathrm{~km} \mathrm{~s}^{-1}$ from Scholz et al. (2004). Therefore, the errors in the $U V W$ space velocity components are dominated by the uncertain distance estimates and by the (partly lacking) $R V$ measurements, whereas the proper motion errors play only a minor role.

We have computed possible heliocentric Galactic space velocities $U V W$ (Johnson \& Soderblom 1987), positive towards the Galactic Centre, Galactic rotation, and North Galactic Pole, separately for the two objects using their positions, the individual proper motions (Table 3, run \#4), and a wide parameter space for the parallaxes (from 10 to 40 mas in steps of 0.5 mas) and $R V \mathrm{~s}$ (from -300 to $+300 \mathrm{~km} \mathrm{~s}^{-1}$ in steps of $5 \mathrm{~km} \mathrm{~s}^{-1}$ ). An exact agreement, to within $5 \mathrm{~km} \mathrm{~s}^{-1}$ for all three $U V W$ velocity components, could only be achieved for positive $R V \mathrm{~s}$, where with distances of the order of $50 \mathrm{pc}$ the $R V \mathrm{~s}$ were required to be larger than $+140 \mathrm{~km} \mathrm{~s}^{-1}$. Generally, the agreement in the space velocities was achieved with slightly larger parallaxes (+1 mas) and $R V \mathrm{~s}\left(+5 \mathrm{~km} \mathrm{~s}^{-1}\right)$ for SSSPM 2003 compared to those of SSSPM 1930.

However, if we consider the large negative $R V$ of SSSPM 1930 measured by Scholz et al. (2004) to be approximately correct, then we can only achieve an agreement in all three space velocity components to within $25 \mathrm{~km} \mathrm{~s}^{-1}$. In this case, SSSPM 2003 would lie at $\approx 32$ pc but SSSPM 1930 would be much too close $(<27 \mathrm{pc})$ compared to our spectroscopic estimate. If we allow for differences in the $U V W$ components of up to $30 \mathrm{~km} \mathrm{~s}^{-1}$, then we get the following solution with individual distances close to our preferred common distance of about $50 \mathrm{pc}$ :

SSSPM 1930: $d=44 \mathrm{pc}, R V=-235 \mathrm{~km} \mathrm{~s}^{-1}$

$(U, V, W)=(-245 \pm 25,-157 \pm 51,+59 \pm 16) \mathrm{km} \mathrm{s}^{-1}$;

SSSPM 2003: $d=50 \mathrm{pc}, R V=-220 \mathrm{~km} \mathrm{~s}^{-1}$

$(U, V, W)=(-219 \pm 24,-183 \pm 66,+89 \pm 15) \mathrm{km} \mathrm{s}^{-1}$.

Opposite to our spectroscopic distances, SSSPM 2003 turns out to be more distant and having a smaller negative $R V$ value than SSSPM 1930 in all solutions with the condition of similar $U V W$ components. An unresolved equal-mass binary status of 


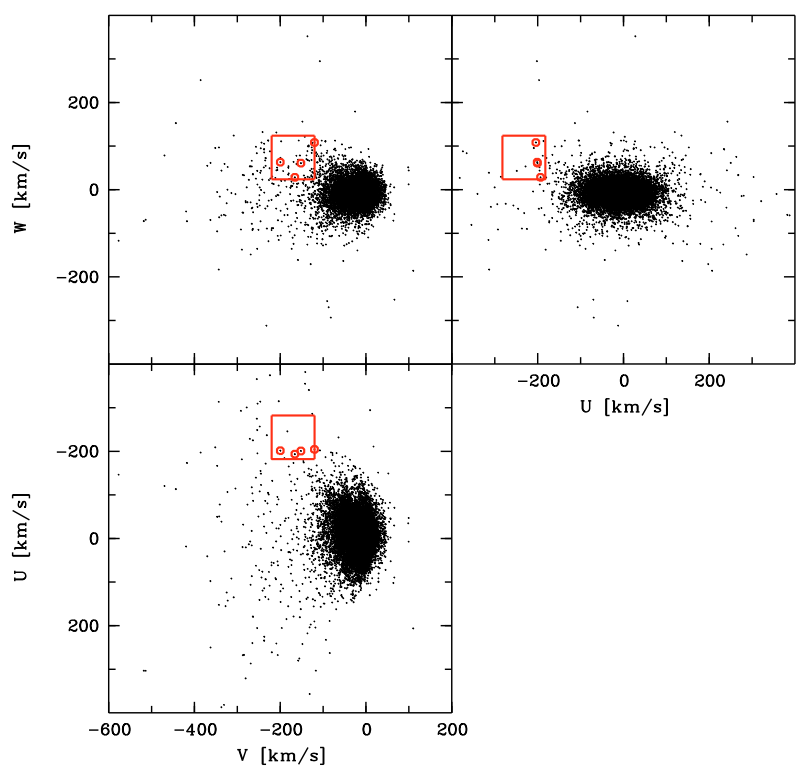

Fig. 4. $U V W$ velocities of 35682 CRVAD-2 stars (dots). Four stars with velocity components within $50 \mathrm{~km} \mathrm{~s}^{-1}$ (squares) of the mean velocity of SSSPM 1930 and SSSPM 2003 are marked (circles).

SSSPM 2003 would lead to a corrected spectroscopic distance of $\approx 54$ pc and help to explain the above mentioned discrepancy.

Although we are aware of the uncertainties in our input data (distances and $R V \mathrm{~s}$ ) and in the assumption of a common space motion of the pair, we can further speculate on the consequences of the computed $U V W$. According to Chiba \& Beers (2000) the above listed large $U$ and $V$ and moderately large $W$ values are more typical of Galactic halo rather than of thick disk stars. To our knowledge, no halo stream with comparable velocity components has been identified so far. The Catalogue of Radial Velocities with Astrometric Data (CRVAD-2; Kharchenko et al. 2007) lists the $R V$ s of about 55000 bright stars together with their proper motions and trigonometric parallaxes if available. We have computed $U V W$ for all CRVAD-2 stars whose parallaxes are more than three times larger than their errors (35682 stars) and searched for stars with velocity components similar (within $50 \mathrm{~km} \mathrm{~s}^{-1}$ ) to the mean $U V W$ of our wide pair (Fig. 4). Only four stars (marked on Fig. 4) were found to satisfy these criteria: Hip 10449, 12294, 18742, 81528. Two of them are in the equatorial zone, the others have large declinations with different signs. The nearest (Hip 10449) is at $\approx 60 \mathrm{pc}$, the others lie between $150 \mathrm{pc}$ and $420 \mathrm{pc}$. Three of these F8- to K0-type stars are widely discussed in the literature as thick disk/halo stars. However, their metallicities are different $(-2.5<[\mathrm{Fe} / \mathrm{H}]<-0.7$ dex, according to the Simbad database $)$.

The similarity of the two subdwarfs LHS 377 and SSSPM 1930 and their clearly different spectra compared to the normal dwarfs (including SSSPM 2003) is well seen in Fig. 3. However, if we apply the procedure proposed by Lépine et al. (2007) to derive revised metallicity classes from the measured spectral indices $\mathrm{CaH} 2, \mathrm{CaH} 3$ and TiO5 of LHS 377 (taken from Gizis 1997) and SSSPM 1930 (from Scholz et al. 2004) we get a $\zeta_{\mathrm{TiO} / \mathrm{CaH}}$ of 0.994 and 0.918 , respectively. Consequently, the two objects would be classified as normal dwarfs (dM) instead of subdwarfs (sdM), M 7.2 and M 6.9, respectively. Unfortunately, their indices fall exactly in the nonvalidated region $(0.2<$ $\mathrm{TiO} 5<0.7,0.5<\mathrm{CaH} 2+\mathrm{CaH} 3<0.9)$ of the classification diagram used by Lépine et al. (2007), where they mentioned a different slope of the iso-metallicity lines obtained from model spectra. Therefore, we prefer our sdM 7 classification of SSSPM 1930, hinting at a lower metallicity compared to the M7 dwarf SSSPM 2003, until better measurements and classification schemes will be available. The metallicity problem does not affect our crude estimates for the distance and space velocity of the pair.

The two objects described in this paper form the widestknown pair of (very low-mass) stars with a large proper motion in common. More detailed investigations of this extreme pair are certainly needed, in particular accurate $R V$ measurements of both components. Trigonometric parallaxes will further improve the velocity components and help to explain the physical association of the objects with each other and their possible membership in a Galactic halo stream.

Acknowledgements. We would like to thank the anonymous referee for his/her comments and suggestions, which helped us to improve the paper. We acknowledge the use of the Simbad database and the VizieR Catalogue Service operated at the Centre de Données astronomiques de Strasbourg (CDS), France. This study was supported by the German DFG grant 436 RUS $113 / 757 / 0-2$, and by the Russian RFBR grant 07-02-91566.

\section{References}

Allen, C., Poveda, A., \& Herrera, M. A. 2000, A\&A, 356, 529

Allen, C., Poveda, A., \& Hernández-Alcántara, A. 2007, IAU Symp., 240, 405

Catalán, S., Isern, J., Carciá-Berro, E., et al. 2008, A\&A, 477,213

Chanamé, J., \& Gould, A. 2004, ApJ, 601, 289

Chiba, M., \& Beers, T. 2000, AJ, 119, 2843

Cutri, R. M., Skrutskie, M. F., Van Dyk, S., et al. 2003, The 2MASS AllSky Catalog of Point Sources, University of Massachusetts and Infrared Processing and Analysis Center (IPAC/California Institute of Technology)

Epchtein, N., de Batz, B., Capoani, L., et al. 1997, Msngr 87, 27

ESA 1997, HIPPARCOS and Tycho catalogues, ESA-SP, 1200

Gizis, J. E. 1997, AJ, 113, 806

Gizis, J. E., Monet, D. G., Reid, I. N., et al. 2000, AJ, 120, 1085

Gliese, W., \& Jahreiß, H. 1988, Ap\&SS, 142, 49

Halbwachs, J. L. 1986, A\&AS, 66, 131

Hambly, N. C., MacGillivray, H. T., Read, M. A., et al. 2001, MNRAS, 326, 1279

Johnson, D. R. H., \& Soderblom, D. R. 1987, AJ, 93, 864

Lépine, S., \& Bongiorno, B. 2007, AJ, 133, 889

Lépine, S., \& Shara, M. M. 2005, AJ, 129, 1483

Lépine, S., Rich, R. M., \& Shara, M. M. 2007, ApJ, 669, 1235

Lodieu, N., Scholz, R.-D., McCaughrean, M. J., et al. 2005, A\&A, 440, 1061

Luyten, W. J. 1940-1987, Publ. Astr. Obs. Univ. Minnesota III, part 3, 35, VizieR On-line Data Catalog: I/130

Luyten, W. J. 1979a, LHS Catalogue: a catalogue of stars with proper motions exceeding $0.5^{\prime \prime}$ annually, University of Minnesota, Minneapolis

Luyten W. J. 1979b, New Luyten Catalogue of Stars with Proper Motions Larger than Two Tenths of an Arcsecond, Univ. Minnesota, Minneapolis

Kharchenko, N. V., Scholz, R.-D., Piskunov, A. E., Röser, S., \& Schilbach, E. 2007, AN, 328, 889

Reid, I. N., Kirkpatrick, J. D., Liebert, J., et al. 2002, AJ, 124, 519

Scholz, R.-D., Lehmann, I., Matute, I., \& Zinnecker, H. 2004, A\&A, 425, 519

Scholz, R.-D., Meusinger, H., \& Jahreiß, H. 2005, A\&A, 442, 311

Seifahrt, A., Guenther, E., \& Neuhäuser, R. 2005, A\&A, 440, 967

Wertheimer, J. G., \& Laughlin, G. 2006, AJ, 132, 1995 